\title{
Die Arbeit an einer EU-Identität
}

\author{
U-Boot-Filme über den Zweiten Weltkrieg: Laconia/The Sinking \\ of the Laconia (D/UK 2001, R.: Uwe Janson)
}

IRINA GRADINARI

\begin{abstract}
Numerous contemporary feature films are working on the creation of a pan-European identity. The genre of war films has been particularly active in this context, attempting to ground the emergence of European identity as arising from the Second World War. The BritishGerman co-production Laconia/The Sinking Of The Laconia (D/UK 2001, R. Uwe Janson) is a symptomatic example of how contemporary political discourses are often justified through an interpretation of the past. This film combines the submarine film with the ship disaster film: the sea, due to its visual resolving power, allows a laboratory space to be created in which, through abstraction and symbolization of what is depicted, transformations in the politics of memory - such as the creation of a new transnational collective - become possible.
\end{abstract}

Title: Submarine Movies About the Second World War: Working on an Identity of European Union in Laconia/The Sinking of the Laconia (D/UK 2001, R. Uwe Janson)

Keywords: Laconia; The Sinking of the Laconia; submarine film; the Second World War; Atlantic Ocean

\section{Aktuelle Umdeutungen nationaler Gedächtnisse}

Das U-Boot-Thema bildet spätestens seit dem Kalten Krieg ein wichtiges filmisches Subgenre des Kriegsfilms (vgl. Koldau 2012), in dessen Rahmen kollektive Erinnerungen der BRD über den Nationalsozialismus verhandelt werden. Der deutsch-britische Fernsehzweiteiler Laconia/The Sinking of The Laconia aus dem Jahr 2011 verarbeitet und aktualisiert daher bereits bestehende Bilder in Bezug auf neue politische Bedürfnisse - die Gestaltung einer gesamteuropäischen Identität. Doch wie soll diese aussehen? Wie kann die Existenz einer solchen Identität ausgerechnet aus der Vergangenheit des Krieges heraus legitimiert werden? Laconia ist in diesem Zusammenhang als symptomatisch wie paradigmatisch zu bezeichnen. Der Fernsehfilm stellt einen tatsächlichen, kaum bekannten Kriegsvorfall dar, nämlich die Rettung der Passagier*innen des britischen Schiffes Laconia im Jahr 1942 durch ein deutsches U-Boot, das dieses Schiff in dem Glauben, es handele sich um ein Militärschiff, zuvor torpediert hatte. Daraufhin wurde durch den Oberbefehlshaber der Kriegsmarine, Admiral Karl Dönitz, am 17. September 1942 der Laconia-Befehl erlassen, der den deutschen U-Booten untersagte, die Schiffbrüchigen versenkter gegnerischer Schiffe 
zu retten (vgl. z.B. Blair 1999), was im Prinzip die Politik der verbrannten Erde an der Ostfront auf den Ozean übertrug. Mit Thomas Elsaesser (vgl. 2006) wäre nun die Frage zu stellen, warum der Film ausgerechnet im Jahr 2011 entstand. Der Fall Laconia geriet eventuell deswegen in >Vergessenheit<, weil er in das weltweit etablierte und über Jahrzehnte ausgehandelte Erinnerungsbild des NS-Regimes nicht recht $\mathrm{zu}$ passen schien. Er verdeutlicht zudem die Widersprüchlichkeit und Heterogenität der Vergangenheit: Es ist nicht möglich, diese nach kausal-logischen Mustern zu erzählen. So ist es auch nicht weiter verwunderlich, dass dieser Vorfall erst zu einem recht späten Zeitpunkt zum Thema eines Spielfilms und somit der breiten Bevölkerung bekannt wurde.

Das historische, ungewöhnliche Ereignis wird durch den Film mit Hilfe einer in Deutschland tradierten Symbolik inszeniert und durch Erzähllogiken und Motive des Hollywood- sowie des englischen Kinos modifiziert. Mit diesem Vorgehen wird das Ziel verfolgt, im Rahmen eines interkulturellen Austauschs von ästhetischen Elementen und Bildern eine konsolidierende, transnationale gesamteuropäische Erinnerung und dadurch die europäische Gemeinschaft selbst performativ herzustellen. Diese transnationale Gemeinschaft muss vor allem aus ihrer Verbundenheit mit dieser kollektiven Symbolik heraus überzeugend wirken. Eine solche Gesamtidentität stellt dabei nicht einfach eine Summe von verschiedenen nationalen Erinnerungen dar, welche offensichtlich die europäischen Länder in ihrer jeweiligen national etablierten Form trennen und keine Einigung auf eine Vergangenheit und somit auf eine gemeinsame Identität ermöglichen. Mit der Inszenierung dieses eher positiven Vorfalls wird hingegen eine Umdeutung der Vergangenheit, nämlich des Verlaufs des Krieges, angestrebt. Deswegen erschien dieser Film gerade rechtzeitig, also gerade in dem Augenblick, in dem das politische Bedürfnis danach entstand. Auf einmal schien es wieder so, als ergäbe sich die Chance, in der Vergangenheit anders zu handeln, den Krieg früher (bevor all die Grausamkeiten stattgefunden hatten) zu beenden und sich mit den ehemaligen Kriegsgegnern zu versöhnen - genau das wird im Film performativ vollzogen. Verwendet werden dafür die jeweiligen tradierten nationalen Symbole, Bilder und Motive, die das kollektive Bildrepertoire im Sinne des kollektiven Gedächtnisses, vielleicht sogar des jeweiligen kollektiven Unbewussten, aktivieren - im Film glaubt man alles schon gehört und gesehen zu haben -, um ein neues transnationales Gedächtnis zu schaffen.

Die Aktualisierung der Vergangenheit für die Gegenwart ist dabei eine spezifische Leistung des Mediums Film, das immer nur aus der Gegenwart seiner Produktion sprechen kann und auch will. In der Gegenwart verbinden die Bundesrepublik Deutschland und das Vereinigte Königreich - aktuellen politischen Entwicklungen zum Trotz - über mehrere Jahrzehnte andauernde, freundschaftliche, kulturelle, ökonomische und militärstrategische Beziehungen, die insbesondere auf die Zeit des Kalten Krieges zurückgehen. Die Tatsache, dass es sich bei dem Film um eine Koproduktion handelt, deutet bereits auf die fruchtbare Beziehung der beiden Länder hin. Vor diesem Hintergrund werden also nationale Vergangenheitsbilder von Deutschland und England, ferner Italien und Polen in der Europäischen Union neu justiert, um daraus eine EU-Identität heraus- 
zubilden und zu legitimieren, die bereits im Szenario des Zweiten Weltkrieges sichtbar wird: Dieser Krieg erscheint nun für die genannten Länder nicht mehr als eine konfliktvolle ideologische und strukturelle Trennung, die sich im Systemwiderstreit zwischen Osten und Westen und so im Kalten Krieg mit veränderter Frontstellung fortsetzte, sondern als die Geburtsstunde der Europäischen Union, die alle (zumindest westeuropäischen) Länder zunächst zum kritischen Reflektieren ihrer Vergangenheit und daran anschließend zur Versöhnung untereinander bewegen kann. Dieses Streben nach Versöhnung besteht generell im deutschen, russischen oder auch im US-amerikanischen Kriegsfilm der Gegenwart und wird in der Regel über das Motiv einer heterosexuellen Liebesbeziehung verhandelt, was teilweise auch in Laconia geschieht. Darüber hinaus erschafft der Fernsehzweiteiler Laconia/The Sinking of Laconia eine transnationale Erinnerungsfigur, die sowohl für das deutsche als auch für das englische Publikum zur Identifikation angeboten wird.

\section{Das Meer als prekärer Erinnerungsraum}

Das Meer besitzt für den Film generell eine besondere Bedeutung und stellt zugleich eine spezifische Herausforderung dar: Trotz der technisch möglich gewordenen Tiefenforschung ist es visuell ein semantisch leerer Raum - »der Ungrund aller Differenzen« (Wolf 2013: 394), der keine Orientierung bietet und daher jegliche Art von Grenzen und Hierarchien auflöst. Zum Beispiel werden im Film Titanic (USA 1997, R.: James Cameron), auf den Laconia mehrfach anspielt, Klassengrenzen überschritten. In Laconia geht es um die Transgression von nationalen Grenzen und somit nationalen Erinnerungskulturen. Zugleich ist das Meer jedoch ein dynamischer und instabiler Raum, der im Gegensatz $\mathrm{zu}$ anderen tendenziell asemantischen Räumen wie etwa dem Wald oder dem Himmel in der Regel selbst zum Akteur wird (vgl. auch Mauer 2010: 13): Es überwältigt die Figuren und bringt so existenzielle Zustände zum Ausdruck, ja jene menschliche Verletzbarkeit und das grundsätzliche Ausgeliefertsein der Umwelt und dem Zufall gegenüber (vgl. z.B. Butler 2010: 22): »Das Meer ist ein gewaltiges Reservoir von Daseinsmetaphoriken« (Wolf 2013: 15).

Das Meer besitzt also eine eigene Materialität und Medialität, die den ästhetischen, technischen und medialen Strategien Widerstand leisten. Deswegen begibt man sich im Film nur selten aufs Meer, um tatsächlich ein Reiseziel zu erreichen. Nicht einmal in historischen Filmen sind Ziele oder Entdeckungen von hoher Priorität: Der Aufenthalt auf dem Ozean oder Meer erscheint oft als erzählstrategischer Selbstzweck, um Figuren in einen Raum zu bringen, in dem radikale Zustandsänderungen, Schwellensituationen und liminale Übergänge möglich werden (vgl. z.B. Ritzer 2010), welche dort gerade durch die sinnauflösende Kraft der See legitimierend wirken. So hat auch Burkhardt Wolf es in der Literatur und im Film beobachtet: 
Mit der Seefahrt geht eine dauernde Neuverhandlung elementarer Grenzen einher, von denen sich dann Basisunterscheidungen wie die zwischen Sicherheit und Gefahr, Aufklärung und Barbarei, Zivilisation und Wildheit oder Kunst und Nicht-Kunst ableiten. [...] Deswegen ist die Seefahrt in mythologischer, religiöser, politischer, juristischer und allgemein kultureller Hinsicht durchweg skandiert von Gründungs- und Entgründungsszenarien. (Wolf 2013: 16)

Ähnlich verhält es sich mit dem Meer in Bezug auf Erinnerungen: Auf See verlieren sich jegliche geschichtlichen Stützpunkte, politischen Orientierungen, topologischen Ordnungen wie topografischen Grenzen, die für die Gestaltung der historischen Erfahrung allgemein und daher auch für das kulturelle und kollektive Gedächtnis insbesondere von zentraler Bedeutung sind. ${ }^{1}$ Konkrete historische Orte bilden einen integralen Bestandteil geschichtlicher Wissenssysteme, stellen selbst Formen von Monumenten dar, die scheinbar die Zeit anhalten und so die Vergangenheit fass- und begehbar machen. Im Film bieten die als historisch gestalteten Räume die Aktivierung des kollektiven Wissens der Zuschauenden an, aber der Film strebt auch nach einer historischen Authentifizierung - so spielen im Film Dokumentationen aus dem Zweiten Weltkrieg oder das Drehen am Ort des realen Geschehens eine zentrale Rolle (vgl. Klein/Stiglegger/Traber 2006; Heller/Röwekamp/Steinle 2007). Das Meer hat hingegen kein evidentes ablesbares Gedächtnis: Es scheint ein zeit- und geschichtsloser Raum zu sein, der zwar eine große Rolle für die Menschheit spielt, sich ihr gegenüber jedoch selbst indifferent verhält, ja vielleicht gerade die Nichtigkeit der historischen Entwicklung der Menschheit hervorhebt (vgl. Assmann 2006).

Warum begibt man sich dann im deutschen Kriegsfilm aufs Meer, wenn sich die Geschichte dort aufzulösen scheint und der Krieg im Atlantik historisch gesehen zudem eine relativ geringe Rolle für den Verlauf des gesamten Krieges spielte? Die Alliierten hatten schon früh den Funkcode entschlüsselt und waren so in der Lage, die deutschen U-Boote aufzuspüren. Das passt natürlich generell zu anderen Kriegsfilmen aus der BRD, in denen die Niederlage einen zentralen Topos darstellt, der bereits von Beginn an die Narration bestimmt. Jedoch erscheint das Filmen auf dem Meer als eine technische und finanzielle Herausforderung. Das Boot (BRD 1981, R.: Wolfgang Petersen) war zum Beispiel mit 25 Millionen D-Mark bis dahin die teuerste Filmproduktion Westdeutschlands. Ist es nun unbedingt notwendig, die Niederlage so aufwendig zu gestalten?

Die U-Boot-Filme weisen also eine erinnerungspolitische Implikation auf. UBoote wurden bereits im Ersten Weltkrieg eingesetzt, auch die ersten deutschen U-Boot-Dokumentarfilme Der magische Gürtel (D 1917, R.: Hans Brennert) und U-Boote heraus! Mit»U-178« gegen den Feind (D 1917, P.: Bild- und Filmamt) sowie der erste deutsche U-Boot-Spielfilm Morgenrot (D 1933, R.: Gustav Ucicky) handeln vom Ersten Weltkrieg (vgl. Grob 2010). Trotzdem beherrscht der Seekrieg

1 Darauf deuten zahlreiche Studien zu Orten, Topografie und Topologie des kollektiven Gedächtnisses hin; vgl. François/Schulze 2001; Weigel 2002; Nora 2005; Csáky/Leitgeb 2009; Döring/Thilmann 2009 und Sabrow 2009. 
weder die Ikonografie des Ersten Weltkrieges noch die Ikonografie des Zweiten Weltkrieges entscheidend. Bekanntlich assoziieren wir den Ersten Weltkrieg eher mit Schützengräben und Gasattacken, den Zweiten hingegen mit Bombenkrieg, Panzerschlachten und Konzentrationslagern. Ende der 1950er Jahre entsteht eine erste Konjunktur von U-Boot-Filmen im Westen. Fast gleichzeitig wurden Haie und kleine Fische (BRD 1957, R.: Frank Wisbar), Duell im Atlantik [The Enemy Below] (USA 1957, R.: Dick Powell), U 47 - Kapitänleutnant Prien (BRD 1958, R.: Harald Reinl) und Die Wölfe in der Tiefe [Lupi nell'abisso] (I 1959, R.: Silvio Amadio) produziert, auf deren Motive und Bilder später der Kultfilm Das Boot zurückgreifen kann (weitere Filme bei Kamps 2006; Koldau 2012). Dieser letztgenannte Film aktualisiert die Ikonografie der 1950er Jahre und dient als Vorlage für Laconia.

Mit den U-Boot-Filmen wird also deutlich, wie diskurspolitisch Kriegsikonografie ist und wie wenig diese allein mit der technischen Entwicklung oder der historischen >Wahrheit< zu tun hat. Das Bedürfnis nach U-Boot-Filmen kommt, so meine These, durch die Veränderungen politischer Konjunkturen auf. Obwohl der Fokus der westdeutschen U-Boot-Filme der 1950er Jahre auf dem Zweiten Weltkrieg lag, verarbeiteten sie mit dem Motiv des Meeres insbesondere den Kalten Krieg, der ein schwer greifbarer, eher abstrakter Krieg der Medienpropaganda und zugleich ein Geheimkrieg der Spionage war (vgl. Horn 2007). Das Meer bietet gerade aufgrund seiner erwähnten spezifischen Materialität und Medialität einen abstrakten Raum an, der keine geografischen Festlegungen verlangt und keinem der Staaten gehört, also einen rein hypothetischen Raum, zum Experimentieren nutzbar, frei von nationaler Symbolik sowie zeitlichen und räumlichen Dimensionen. Aus diesem Grund fördert das Meer wiederum das Modellhafte; so verdichtet es den Krieg zum Spiel der Maschinen und der Technologien - geht es doch im Kalten Krieg um die Demonstration technischer Überlegenheit. Das Meer codiert die Kriegsführung in eine technische Arbeit - zum Beispiel Abhören oder Apparate bedienen - um, die zum Medialen des Kalten Krieges viel besser passt. Außerdem sind die U-Boot-Filme dazu geeignet, ehemalige Feinde im Unklaren zu halten. Sie werden in den westdeutschen Filmen weder gezeigt noch genauer thematisiert. Torpedierte Schiffe sind nur für einen kurzen Zeitraum am Horizont zu sehen, in der Regel durch Periskope (Abb. 1-2).

Großbritannien, Hauptgegner des Dritten Reichs im Atlantik, ist in den 1950er Jahren der Nato-Partner und Alliierte Westdeutschlands gegen die Staaten des Warschauer Paktes. So erlauben es die U-Boot-Filme, Feindschaft so diffus wie möglich zu gestalten, zugleich jedoch die BRD als technische und militärische Weltmacht über die »Magie der Technik « (Wolfgang Petersen in einem Interview bei Rosner 1981) zu erleben, ohne die NS-Symbolik reproduzieren zu müssen: Die Ikonografie des U-Boot-Films braucht keine etablierten Bilder des kollektiven Gedächtnisses über den Zweiten Weltkrieg, löst sich so von den meisten NS-Symboliken und Motiven ab und scheint wegen ihrer Technizität auch jenseits des Ideologischen ins Reich der nackten Faktizität der Maschinen zu gelangen. 
Abbildungen 1 und 2: Haie und kleine Fische. Der Gegner wird durch optische Medien in die Ferne gerückt. Außerdem wird hier ein zeitlicher Abstand zwischen der gespielten Szene in den 195oer Jahren und der eingeblendeten Dokumentation aus dem Krieg - das sieht der Kapitän im Periskop - installiert.
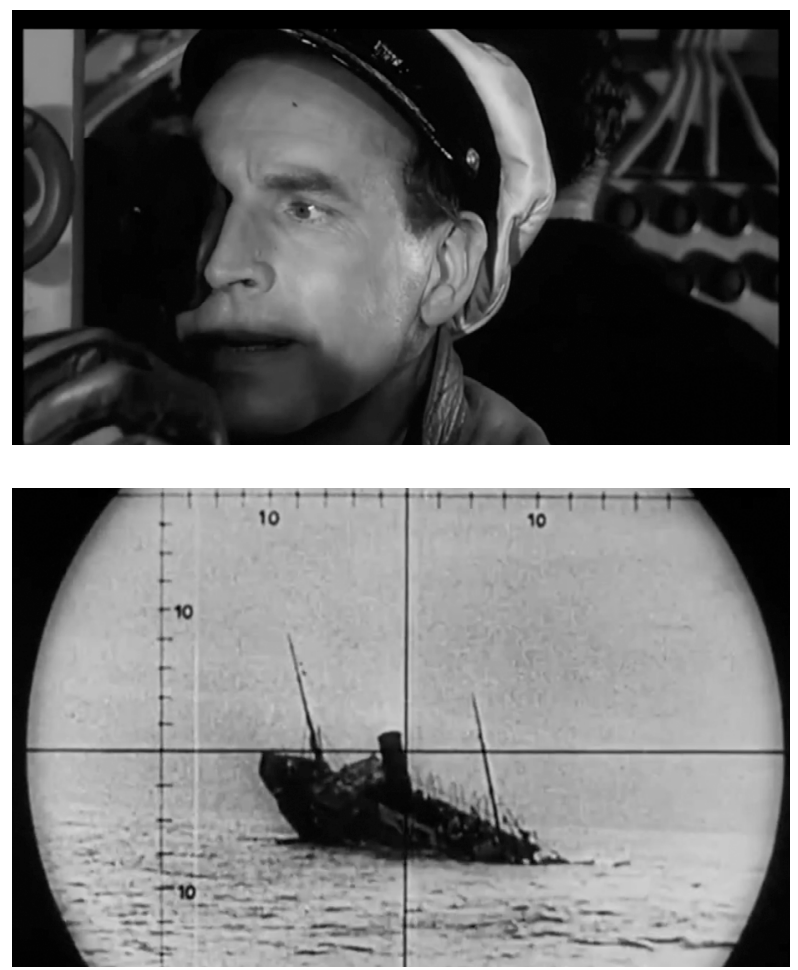

Die Auflösungskraft des Meeres bewirkt jedoch noch mehr: Gerade aufgrund des Mangels an zeitlicher und räumlicher Kontextualisierung, die für den Kriegsfilm konstitutiv ist, wird die Handlung vom Konkreten ins Symbolische entrückt. Der U-Boot-Film ermöglicht es daher, am Beispiel eines U-Bootes den ganzen Krieg durchzuspielen, seine Quintessenz zum Ausdruck zu bringen und dabei vor allem eine besondere Form des Kollektiven zu verhandeln, was auch für Laconia von entscheidender Bedeutung ist. In U-Boot-Filmen geht es, so möchte ich meine These fortführen, darum, die Herausbildung eines nationalen wehrhaften Kollektivs, das allein als männlich inszeniert wird (vgl. auch Koldau 2012), $\mathrm{zu}$ demonstrieren, $\mathrm{zu}$ analysieren oder gar performativ zu vollziehen. Dazu gehören vor allem die Szenen der Integration neuer Seekadetten in die U-BootMannschaft, die nie reibungslos verläuft. So beginnt Haie und kleine Fische mit einem Streit zwischen der bestehenden Mannschaft und einem neuen Matrosen jüdischer Herkunft (wobei dies im Film als Phantasma gezeichnet wird). Eine ähnliche Szene einer konfliktvollen Integration eines neuen Matrosen gibt 
es auch in Laconia, in der nun die Bedeutung der Solidarität auf dem Meer für das Überleben hervorgehoben wird. Im Film Das Boot bekommt der Reporter einen schmutzigen, verölten Lappen ins Gesicht, was seine Nichtzugehörigkeit zur Bootsmannschaft zum Ausdruck bringt und somit die Zuschauenden, zumindest vorerst, auf Distanz zur Mannschaft hält. Solche Strategien erschaffen ein Kollektiv, in das auch das Publikum durch die Figur des Neulings Schritt für Schritt integriert wird. Zur performativen Erschaffung des wehrhaften Kollektivs gehören zudem die Totalen auf das U-Boot mit der Mannschaft an Deck, bei denen die Menschen nun als eine Einheit gegenüber allen Gefahren präsentiert werden (Abb. 3-4).

Abbildung 3: Haie und kleine Fische. Das deutsche U-Boot als Einheit.

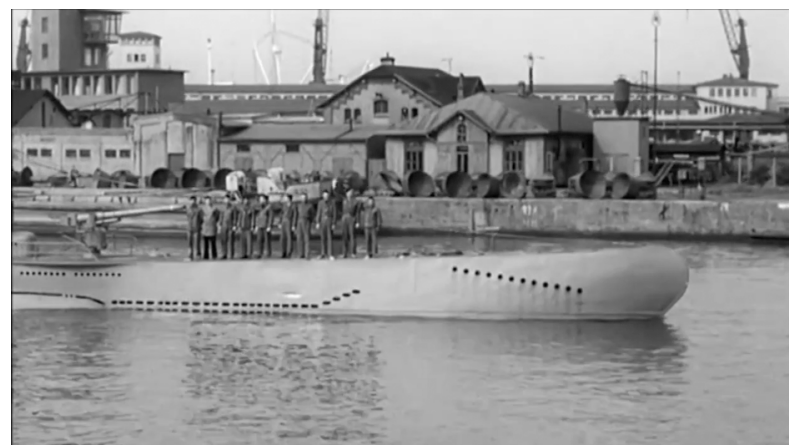

Abbildung 4: Laconia. Das deutsche U-Boot mit den Flüchtlingen an Deck und den Rettungsbooten wird durch die Totale als Einheit etabliert.

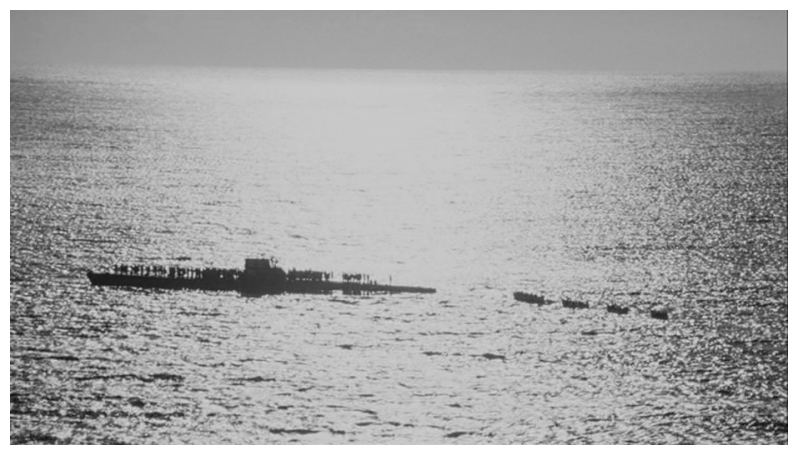

Zentral sind letztendlich Szenen, die die Mannschaft im U-Boot selbst als Kollektiv oder bei der gemeinsamen Arbeit als Bestandteil einer Maschine, ja gar als eine Vereinigung mit ihr, vorführen. Die Funktionsweise des U-Bootes ist auf eine gemeinsame, reibungslose Arbeit angewiesen, bei der die Matrosen in ihrer Arbeitsteilung als Bestandteile eines Organismus oder sogar eines Mecha- 
nismus inszeniert werden. Wenn das U-Boot angegriffen wird, kommt es immer zur Verletzung eines der Mannschaftsmitglieder - so wird die Haut des Kollektivkörpers durch den Korpus des U-Bootes gebildet (Abb. 5-6). Für den Film Laconia ist es beispielsweise von zentraler Bedeutung, dass das U-Boot eine abstrakte Kollektivität performativ erzeugen kann: Relativ am Anfang der Handlung werden auch hier unterschiedliche Abteilungen des Bootes von der sich durch den Raum bewegenden Kamera etabliert, wodurch deutlich wird, dass das einzelne Individuum nicht länger zählt, sondern nur Teil der Maschine ist, während das englische Schiff zunächst in sich heterogen bleibt.

Abbildung 5: U 47 - Kapitänleutnant Prien. Das U-Boot als Einheit im Inneren.

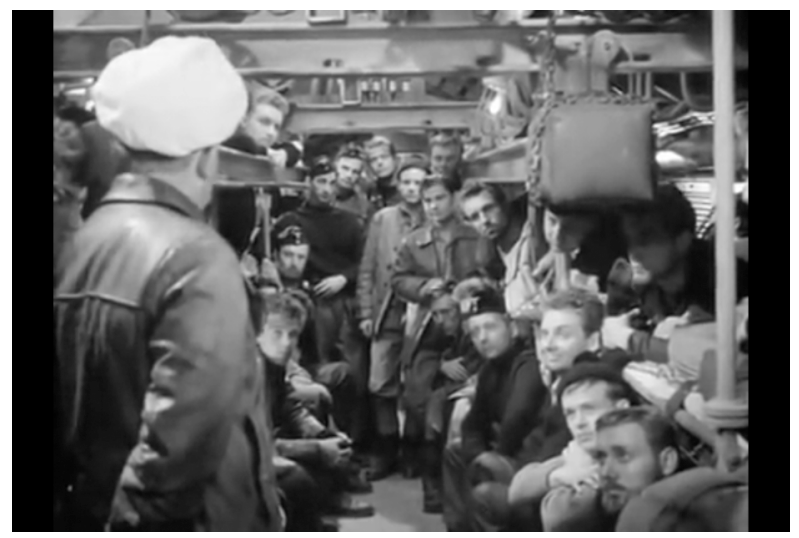

Abbildung 6: Das Boot referiert auch auf die ikonografische Tradition, im Inneren eine solidarische Kollektivität zu erzeugen.

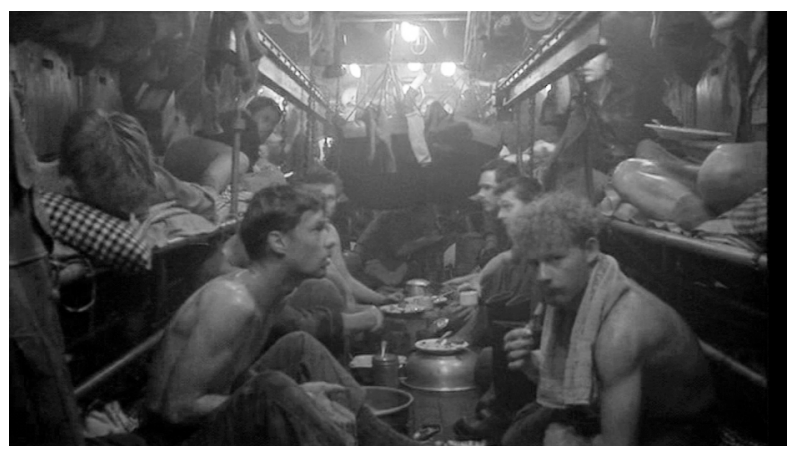

Westdeutsche U-Boot-Filme der $1950 e r$ und 1980er Jahre verhandeln dabei jene Form von Kollektivität, die einerseits politisch, andererseits jedoch vom NS-Staat abgekoppelt ist. Zwar werden die Innenräume des U-Bootes mit anderen Räumen auf dem Land - in Laconia etwa mit dem NS-Militärstab in Paris, mit dem 
englischen Kommandopunkt in Afrika und einem US-amerikanischen Militärstab - durch den Schnitt verknüpft, doch repräsentieren die U-Boot-Filme über den Zweiten Weltkrieg nicht die jeweiligen Staatsideologien, sondern allein jene essenzialistisch anmutenden Kollektivkerne der Nationen, die dann im Angesicht existenzieller Herausforderungen von den jeweils aktuellen politischen Devisen abweichen und einander in ihrer Andersheit zugleich ähnlich werden. Die Tiefseebilder verstärken diesen Eindruck, sich im kollektiven Unbewussten zu befinden und dieses irgendwo an der Peripherie und in der Tiefe zu bereisen. Die Abkoppelung findet buchstäblich in der Handlung statt - die Verbindung mit dem Land wird unterbrochen, die Befehle kommen zu spät oder gar nicht mehr an, und so müssen die Figuren selbstständig handeln, um zu überleben. Die meisten sind sowieso eher dazu gezwungen, sich am Krieg zu beteiligen, die NS-Anhänger (ausschließlich Männerfiguren) sind in der Regel in der deutlichen Minderheit. Langsam werden auch sie immer kritischer gegenüber der NS-Ideologie. Laconia spitzt diese politische Abkoppelung durch die Rettung der Schiffbrüchigen noch einmal zu, die sogar allgemein der Kriegslogik widerspricht und eigentlich einen humanistischen Akt mitten in der Zerstörung darstellt. Im Gegensatz zu den U-Boot-Filmen des Kalten Krieges über die atomare Bedrohung, die in diesem Heft Johannes Pause ausführlich behandelt, sind die U-Boote im Kontext des Zweiten Weltkrieges eben keine »Staatsschiffe« (Wolf 2013: 187), sondern das als substanziell und technisch inszenierte Wunschbild von einem Kollektiv, ja sein in der Regel ideologisch verdeckter und nun bloßgestellter Kollektivkern, der allein aus dem Begehren nach Zusammenhalt, Pflichterfüllung, Solidarität und Hilfsbereitschaft besteht und sich somit angeblich jenseits aller Staatsideologien befindet. Diese Themen kommen auch unmittelbar in den Filmen selbst in Dialogen und Handlungen der Figuren zur Sprache. Die Abkapselung von der NS-Ideologie ist dann auch deshalb notwendig, weil es um identitätsstiftende Kollektivbilder der 1950er Jahre geht - also darum, wie die deutsche Gesellschaft in der Gegenwart der Filmproduktion ist oder zumindest sein sollte - und nicht etwa um das Nachempfinden des NS-Kollektivs.

Daher nimmt diese Kollektivität bzw. dieser nackt geschälte Kollektivitätskern jene psychoanalytisch begründete Subjektivitätsform an, die Klaus Theweleit in den 1970er Jahren in Anlehnung an Norbert Elias (vgl. 1991: 332) als Körperpanzer beschreibt. Theweleit analysiert vor allem die Literatur der Männer in den Freikorps, welche sich einer gewaltsamen Sprache bedient. Der Blick der faschistischen Männer schweift als eine Art Suchscheinwerfer herum, bis er eine Bewegung bemerkt, diese heranzoomt und das Objekt vernichtet: »Statt mit Kamera und Schneidetisch (mit lebendigem Bild und Montage) arbeiten sie mit Scheinwerfern und Hackmessern (mit totem Bild und Zerstückelung).« (Theweleit 1977: 271) Diese Metapher setzen die U-Boot-Filme in Szene, indem sie den Blick mit der Waffe kurzschließen: Die U-Boote suchen mit ihren Teleskopen andere Schiffe, zielen auf sie und vernichten sie.

Der Körperpanzer konstituiert sich dabei über eine Ausgrenzung oder gar Vernichtung der Anderen (vgl. ebd.). Die Anderen, vor allem Frauen, werden bekanntlich bildhistorisch mit Fluten und Strömen unterschiedlicher Art (Blut, 
Schlamm, Sumpf oder auch Geldströmen) assoziiert, welche die männliche Subjektivität zu überschwemmen drohen. Sie verkörpern zugleich ein verdrängtes unbewusstes Begehren, im U-Boot-Film das verdrängte Wissen, das sich als unheimliches Wissen über die NS-Ideologie manifestiert. Das Meer bekommt in mindestens einer der Szenen immer ein menschliches >Gesicht<. In Haie und kleine Fische rettet sich die gegnerische Mannschaft mit einem Rettungsboot, wobei die Szene nur für ein paar Sekunden eingeblendet wird (Abb. 7). In U $47-$ Kapitänleutnant Prien schwimmen im Meer die Leichen der jüdischen Flüchtlinge des gerade versenkten Schiffes, wovon der Held aus dem Filmtitel nichts wissen wollte (Abb. 8). In Das Boot versagt der Kommandant (Jürgen Prochnow) den ertrinkenden Gegnern, die allein am Horizont zu sehen sind, seine Hilfe, was eine der emotionalsten Szenen des Films darstellt (Abb. 9).

Abbildung 7: Haie und kleine Fische. Eine rätselhafte Szene: Die Rettung der Mannschaft eines feindlichen Schiffes wird nur für ein paar Sekunden eingeblendet.

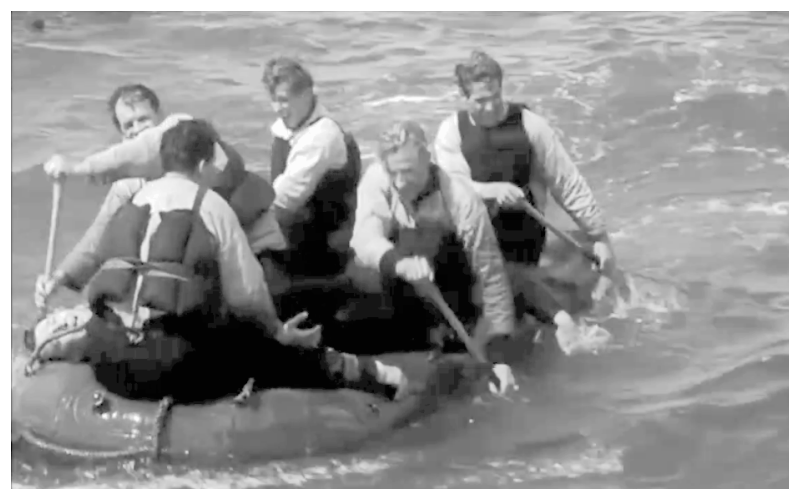

Abbildung 8: U 47 - Kapitänleutnant Prien. Das Meer konfrontiert die Mannschaft mit dem >verdrängten < Wissen der NS-Vernichtungsideologie.

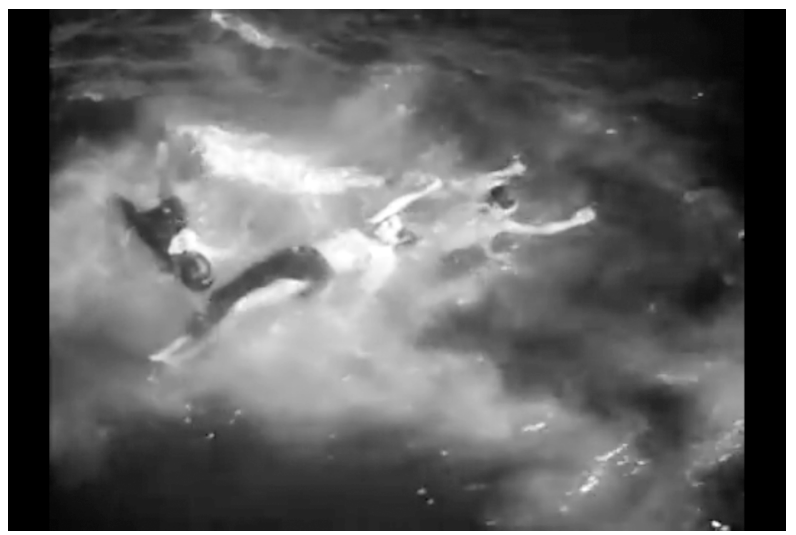


Abbildung 9: Das Boot. Die Gegner erscheinen am Horizont und werden nicht gerettet.

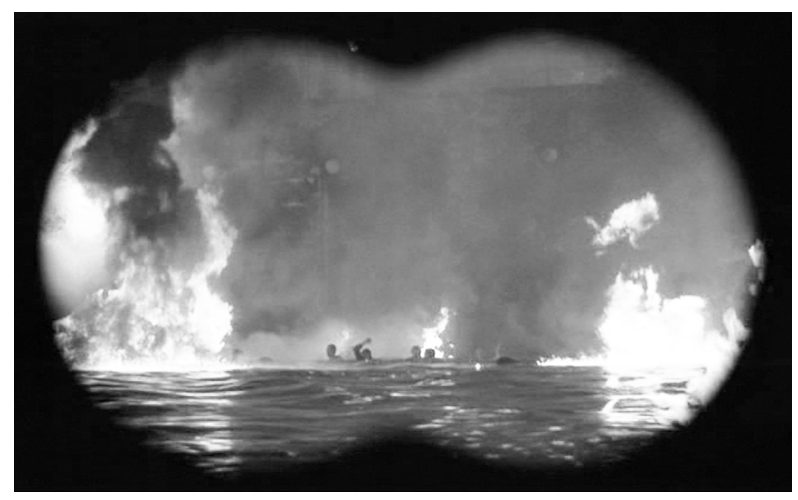

Der Körperpanzer richtet sich nach Theweleit phallisch gegen diese Ströme aus, und innerhalb der männlichen Ordnung fließt uneingeschränkt das Begehren, imaginiert als Begehren der Fülle und des Nichtkastriertseins, wie es beispielsweise in soldatischen Aufmärschen zum Ausdruck kommt. »Der Faschismus übersetzt so innere Zustände in riesige äußere Monumente, Ornamente als Kanalisationssysteme« (ebd.: 550), die in Filmen um den heldenhaften Opfermythos kreisen. Auch in den U-Booten herrschen Erotik und männliche Solidarität vor, in ihrer uhrwerkartigen gemeinsamen Arbeit schließen sie sich zusammen für den Schlag gegen den Feind und - begründet durch den spezifischen Raum - gegen jegliche Fluten. Die Grenzen werden zwischen Ausschweifungen mit Frauen im Bordell auf dem Land und der Disziplin und Enthaltsamkeit auf dem U-Boot, zwischen der solidarischen Arbeit der Mannschaft und der entgrenzenden Kraft des Meeres gezogen. Die Voraussetzung für die Entstehung eines solchen männlichen U-Boot-Kollektivs ist also der Verzicht auf Frauen, die Ermordung von Juden sowie die Vernichtung der am Horizont erscheinenden Gegner. Zugleich ist dieser phallisch aufgeregte Exzesszustand des Männlichen nicht von Dauer. Das lässt sich insbesondere im Hinblick auf die $1950 e r$ und 1980er Jahre sagen, in denen das Männlichkeitsideal durch einen bürgerlichen Familienvater (und nicht etwa eine Militärmaschine) dargestellt wird. Der Körperpanzer ist daher ein technisch ruhmvoller, jedoch einsamer und nicht existenzfähiger kollektiver Zustand, der in dieser Form nur in Gefahrzeiten aus dem Unbewussten mobilisiert werden kann. So geht er immer wieder unter die Versenkung oder die Zerstörung des U-Bootes, allerdings nicht die des Männerkollektivs, gehört zur Spannungsdramaturgie dieser Filme. Visuell-ästhetisch wird die extreme Opposition zwischen dem Weiblichen und dem Männlichen, die Männerbünde auszeichnet (vgl. Widdig 1997: 235), nie aufgelöst, geschweige denn die Begegnungen mit den Gegnern. Entweder sterben die Matrosen, und lassen ihre Frauen allein zurück, oder sie überleben als eine kleine Gruppe, ohne jedoch die Frauenfiguren einzubeziehen (Abb. 10). Vielmehr werden jene Treue zur Führerpersönlichkeit und die Bereitschaft, Opfer zu bringen (vgl. ebd.: 236), 
sowie Kompromisslosigkeit, Aggressivität und Endzeitstimmung (vgl. ebd.; auch Völger/Welck 1990) bestätigt. So erscheinen die Fluten und die Anderen doch als (diffuse, kaum definierbare) Bedrohung, die die phallische Abwehr als existenziellen Kampf legitimiert.

Abbildung 10: Haie und kleine Fische. Das männliche Kollektiv überlebt gerade dank seiner Solidarität und Abwehrkraft.

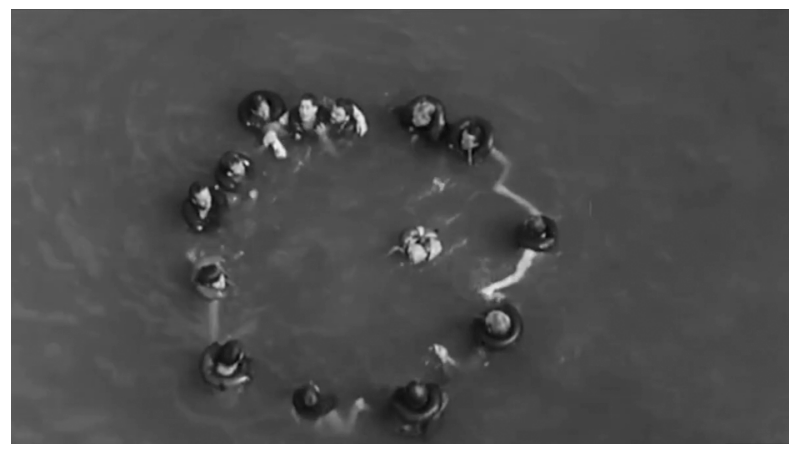

\section{Kulturelle Erinnerungsdifferenzen Und Erschaffung EINER TRANSNATIONALEN ERINNERUNGSFIGUR}

Seit ca. den 200oer Jahren drehen die meisten Filmschaffenden Kriegsfilme mit Schauspieler*innen aus den jeweiligen Ländern der Alliierten. Laconia (deutscher Titel) bzw. Sinking of Laconia (englischer Titel) ist ein Beispiel einer solchen internationalen Zusammenarbeit. Der Fernsehzweiteiler entstand unter der Regie des aus Deutschland stammenden Uwe Janson nach dem Drehbuch eines bekannten britischen TV-Drehbuchautors, Alan Bleasdale, und mit englischen, schottischen, irischen, deutschen, italienischen und polnischen Darsteller*innen in den jeweiligen historischen Rollen von nationalen Akteur*innen. So ist die Filmproduktion selbst ein Politikum. Sie demonstriert einerseits einen Versöhnungsakt durch die Zusammenarbeit, indem nun die dritte und vierte Generation ehemaliger Kriegskontrahenten gemeinsam an der Vergangenheit arbeiten und diese als Fiktion durchspielen. Andererseits hat eine solche nationale Koproduktion eine stark konsolidierende und legitimierende Wirkung. Das Vergangenheitsbild wird nun von beiden oder mehreren Seiten abgestimmt und liegt so scheinbar jenseits des Ideologischen der jeweiligen Nationen - als würde das Geschehen nun tatsächlich so erzählt, wie es >war«!

Der Zweiteiler Laconia ist dabei insofern auch für die Analyse interessant, als er in zwei verschiedenen Fassungen - für das deutsche und das englische Publikum - erschienen ist. Insbesondere bezüglich des ersten Teils, der die Figuren einleitet und die Schiffbruchkatastrophe zeigt, unterscheiden sich die Teile recht stark. Die jeweils ersten Teile holen das Publikum dort ab, wo es sich 
bildästhetisch und erinnerungspolitisch befindet. Auch die Handlung und die Rollen der Figuren sind unterschiedlich. Der zweite Teil bildet hingegen eine gemeinsame europäische Erinnerung jenseits politischer, klassenspezifischer oder historischer Differenzen ab. Er unterscheidet sich in den beiden Fassungen nur minimal. Hier solidarisieren sich die Figuren und sind dann auch alle zusammen gegen Ende des Films buchstäblich in einem Rettungsboot auf dem offenen Meer, das auch als Anspielung auf Lifeboat (USA 1944, R.: Alfred Hitchcock) zu verstehen ist. Der Film stützt sich somit auf die Filmtraditionen beider Staaten - die des deutschen U-Bootes und die des englischen Rettungsboots -, die die Erinnerungsperspektive der jeweiligen Länder von der Seite des Gegners her vervollständigen.

Die deutsche Version beginnt mit der deutschen Figur Hildegard Schmidt, die von der bekannten deutschen Schauspielerin Franka Potente dargestellt wird. Sie befindet sich auf der Flucht und nimmt zu Beginn der Handlung als Hilda Smith eine englische Identität an. Aus Ägypten möchte sie mit dem englischen Schiff Laconia, das erst durch den Suezkanal und dann um den afrikanischen Kontinent herum durch den Atlantik fahren soll, nach Liverpool gelangen. Es wird sofort erklärt, dass sie vor den Nazis flieht, während ihre Identität in der englischen Version einer Spannungsdramaturgie unterliegt und erst etwa in der Mitte des zweiten Teils erläutert wird, warum sie aus Deutschland geflohen ist. Ein kurzes Flashback über die Verhaftung ihrer Familie fehlt in der englischen Fassung ganz. Die englische Version führt hingegen die englische Figur Mortimer, einen jungen Seeoffizier auf dem Schiff Laconia, der von dem bekannten britischen Schauspieler Andrew Buchan verkörpert wird, im Kreis seiner Familie ein. Er feiert zusammen mit seiner Ehefrau und zwei kleinen Kindern den Geburtstag der Mutter. Diese Szene fehlt wiederum in der deutschen Version sie kommt verkürzt in einer Rückblende als Erinnerung Mortimers vor, als dieser vom Tod seiner Familie durch eine deutsche Bombe erfährt. Für das jeweilige Publikum bedeutet dies eine Annäherung an das Andere über die eigene nationale Identifikationsfigur.

An dieser Stelle wirken bereits die Geschlechterdifferenzen. Die Kriegserinnerungen sind in den meisten Ländern an eine männliche Kämpfer- und Soldatenfigur gebunden, die ihr eigenes Land verteidigt und das nationalsozialistische Regime zerstört. Der Seeoffizier Mortimer ist jene mutige Kämpferfigur und dazu ein edler Gentleman, der bei keiner Situation die Fassung und den Anstand verliert. Auf der deutschen Seite werden zwei Figuren angeboten, die zugleich verschiedene Spaltungen im deutschen kollektiven Gedächtnis repräsentieren. Einmal geht es darum (auch genrebedingt durch zahlreiche Kriegsfilme und Wochenschaudokumentationen), die NS-Aggression zu verarbeiten. Diese wird durch ein in früheren Kriegsfilmen ästhetisch ausgehandeltes Figurenset zum Ausdruck gebracht, das die NS-Ideologie von der Hauptfigur abspaltet und so die Figur in ein gegenwärtiges Ideal verwandelt. Hier präsentiert der U-Boot-Kapitän Werner Hartenstein (Ken Duken) jene Erinnerungsfigur, die die historische Person spielt, im Film jedoch nicht den Nationalsozialismus verkörpert. Diese Figur wird als humanistischen Werten verpflichtet gezeigt, obwohl 
sie zunächst als ein vorbildlicher NS-Soldat auftritt, der den Befehlen des Oberbefehlshabers Karl Dönitz (Thomas Kretschmann) zumindest bis zum Fall Laconia immer folgt. Die nationalsozialistische Gesinnung wird von ihm abgespalten. Träger der NS-Ideologie ist einerseits einer der Offiziere von Dönitz im Militärstab (nicht einmal Dönitz selbst) und andererseits der leitende Ingenieur Rostau (Matthias Koeberlin) auf dem U-Boot. Er steht häufig in Opposition zum Kapitän und besitzt ein Tuch mit dem Spruch: »Vergiß nicht, dass Du ein Deutscher bist«, das ihm sein Vater, der wiederum im Ersten Weltkrieg gekämpft hat, vererbt hatte. Dieses Tuch schenkt er seinem Kapitän während der Rettungsaktion der Schiffbrüchigen, womit er sein Nichteinverständnis mit diesem Akt zum Ausdruck bringt. Dadurch wird zugleich deutlich, dass sowohl die nationalsozialistische als auch die nationale Grenze gewaltsam sind - beide fördern eine Logik der Vernichtung. Die Männerfiguren, die diese Logik umsetzen, müssen daher, zumindest symbolisch, verschwinden - sie dürfen keinesfalls eine Zukunft haben und so zum Aufbau der Nachkriegsgesellschaft beitragen, wie es in den deutschen Kriegsfilmen der Gegenwart - etwa in Der Untergang (D 2004, R.: Oliver Hirschbiegel), Dresden (D 2006, R.: Roland Suso Richter) oder Anonyma - eine Frau in Berlin (D/P 2008, R.: Max Färberböck) - der Fall ist. Der Abspann von Laconia informiert dann etwa darüber, dass das deutsche U-Boot im März 1943 mit der gesamten Mannschaft gesunken ist. Auch der englische Offizier Mortimer verschwindet - er gerät in deutsche Gefangenschaft -, obgleich eine mögliche zukünftige Beziehung zwischen ihm und Hilda Smith angedeutet wird.

Aus diesem Grund gibt es eine zweite deutsche Erinnerungsfigur - Hildegard Schmidt bzw. Hilda Smith -, die als die einzige und eigentliche transnationale Identifikationsfigur angeboten wird. Diese Figur referiert nicht nur auf die Europamythen und die ikono- und kartografische Tradition, Europa als Frau darzustellen (vgl. z.B. Bruns 2009), sondern erscheint auch im Kriegskontext als eine pazifistische Figur, die darüber hinaus noch als (Gottes-)Mutter - eine Frau mit Baby - präsentiert wird. Auch diese Erinnerungsfigur wurde in den deutschen Nachkriegsfilmen entwickelt. Bereits im ersten DEFA-Film Die Mörder sind unter uns (DEFA 1946, R.: Wolfgang Staudte) markiert die Frau einen neuen Anfang, der deswegen möglich ist, weil die Frau nicht in die NS-Ideologie verstrickt war. So verkörpert Hilda wenn nicht den Widerstand, so doch zumindest das Nichteinverständnis mit dem NS-Regime. Vor allem hat sie Deutschland noch rechtzeitig verlassen und sich somit topografisch von der NS-Ideologie entfernt. Sie überlebt die Schiffskatastrophe mit einigen englischen Schiffbrüchigen.

Es bestehen weitere kleine Differenzen und Verschiebungen in der Darstellung - unterschiedliche Figuren werden verschieden gewichtet -, wodurch die Fassungen für das jeweilige Publikum eine unterschiedliche Erinnerungsfunktion erfüllen, weshalb sie auch verschieden wahrgenommen wurden. In Großbritannien wurde der Film mehrfach gelobt - die Versöhnung scheint erwünscht und im Film gelungen zu sein. Deutsche Kritiken fielen hingegen ambivalent bis negativ aus. Der Filmkritiker Willi Winkler echauffierte sich beispielsweise 
in der Süddeutschen Zeitung über die deutsche Fassung: »So scheitert das große Euro-Versöhnungswerk ganz wie bei den Großen am partikularen Kleingeist.« (Winkler 2011) Vor allem wurde die deutsche Version mit Off-Kommentaren der deutschen Hauptfiguren versehen, offensichtlich um, soweit möglich, Eindeutigkeit zu produzieren. Sie verdoppeln das Gesehene und legen zugleich das Visuelle fest, das im Grunde für verschiedene Deutungen viel offener ist. Dadurch werden auch die Figuren politisch klar positioniert. Diese Veränderungen müssen als politisch verstanden werden - sie verraten jene etablierte kulturelle Angst in der BRD, das Thema des Krieges zu unterhaltsam zu gestalten, und weisen auf eine lang gehegte Auffassung im deutschen Kino hin, der zufolge Kriegsfilme keinen Genuss bereiten dürfen. Deswegen wirken die Kommentare didaktisch moralisierend - sie lenken die Rezeption stark in eine Richtung, die definitiv den Nationalsozialismus verurteilen soll, und verderben zugleich zum Teil das Kinoerlebnis.

Die englische Fassung erzählt außerdem im Gegensatz zur deutschen kontinuierlich ohne Flashbacks. So zeichnet sich die deutsche Version durch eine Traumastruktur des Erzählens aus, indem beide, die deutsche und die englische Hauptfigur, mit Backstory Wounds (vgl. Krützen 2004: 30) versehen werden, wodurch zugleich das Thema der Erinnerung im Allgemeinen aufgeworfen wird. In der deutschen Fassung sagt Hilda an einer Stelle, dass es besser wäre zu erzählen als zu verdrängen und dass sie gar nicht vergessen könne - Worte, die unverkennbar einen gegenwärtigen Erinnerungsimperativ wiederholen. Sie werden in die Vergangenheit verlagert und erscheinen daher anachronistisch und gewissermaßen selbst als eine Wiederkehr des Verdrängten. Es ist zu erwarten, dass diese Worte dem deutschen Publikum vertraut sind und eine Art Déjà-vu erzeugen, tauchen sie doch auch in anderen erinnerungspolitischen Kontexten auf. Flashbacks, Déjà-vus und Off-Kommentare, die das Innere der deutschen Figuren offenbaren, deuten so die Handlung in eine psychische (Erinnerungs-) Landschaft um.

Letztendlich ist die deutsche Fassung etwa sieben Minuten länger als die englische. Vor allem die Schiffskatastrophe wird für das deutsche Publikum viel ausführlicher und detailreicher dargestellt. Möglicherweise soll das englische Publikum weniger von der Katastrophe sehen, um den Deutschen dann am Ende des Filmes verzeihen zu können. Dort überlebt auch eine Frauenfigur, die am Ende des Films auf einem französischen Rettungsschiff ihre Mutter wiedertrifft (in der deutschen Fassung aber nicht). Das deutsche Publikum muss jedoch erst seine Schuld am Ausmaß der Katastrophe erspüren und miterleben, um dann den Verzicht auf die nationale Gemeinschaft zu akzeptieren. Im ersten englischen Teil werden daher die Doppelmoral der englischen Gesellschaft sowie Klassendifferenzen viel stärker thematisiert, während die deutsche Fassung hier schon affektiv auf ein transnationales Gemeinschaftserlebnis hinarbeitet. Hilda Smith singt das während des Zweiten Weltkrieges populäre britische Lied We'll Meet Again aus dem Jahr 1939, das für sie eine Art Integrationsprobe darstellt - wird sie nun deswegen hinsichtlich ihrer deutschen Identität auffliegen oder nicht? Das Lied war ein Mega-Hit und ist heute ein fest eta- 
blierter Erinnerungstopos der kollektiven englischen Kriegserinnerungen und vielleicht mit dem deutschen Lied Lili Marleen vergleichbar, was der Film selbst unterstreicht: Jede*r kenne doch den Text dieses Liedes. Hilda Smith tritt, gezwungen durch das englische Publikum auf dem Schiff, anstelle der berühmten englischen Sängerin Vera Lynn auf, die dieses Lied vor und mit Soldaten gesungen hat. So wiederholt diese Szene jenen englischen Erinnerungstopos bzw. stellt ihn im Sinne eines Reenactments nach und wird zugleich durch die Frage, ob Hilda Smith die kulturelle englische Erinnerungsikone ersetzen und so zur transkulturellen Erinnerungsfigur werden kann, zu einer Erinnerungsprobe. Ihr Outfit ähnelt dem der Sängerin; die Figur verwandelt so das nationale Motiv in ein internationales. In der englischen Fassung wird das Lied mehrfach durch Parallelhandlungen unterbrochen, es ist zum größten Teil nur im Hintergrund $\mathrm{zu}$ hören. In der deutschen Fassung wird es nur einmal kurz unterbrochen und als ein Ereignis dargeboten, das nun vom deutschen Publikum affektiv erlebt werden kann. Nichts verbindet stärker und affektiver als Musik. Deutsche Zuschauende können es - ähnlich wie das Publikum im Film, das die Haltung der Zuschauer*innen intradiegetisch vormodelliert - mitsingen und sich so an die englische Erinnerungskultur anschließen. Für das englische Publikum soll die deutsche Hilda Smith also nur den bekannten Erinnerungstopos aufrufen und durch die deutsche Schauspielerin aktualisieren, für das deutsche hingegen einen solchen Erinnerungstopos erst herstellen und legitimieren.

\section{Europäische Gemeinschaft AUS DER VERgangenHeIt heraus}

Vor dem Hintergrund verschiedener kultureller Erinnerungsdiskurse möchte der Film eine gemeinsame europäische Kollektividentität performativ herstellen, die zunächst durch die Koproduktion, daraufhin durch eine gemeinsame Vergangenheitsreflexion im Film und letztendlich durch ein gemeinsam erlebtes (Kino-)Ereignis in der Vergangenheit vollzogen wird. Im Zentrum steht vor allem das Ereignis, das die Zerstörung im Krieg mit der Rettung wiedergutzumachen und sich über das Menschliche, etwa durch Sympathien und Liebe, aber auch als Schicksalsgemeinschaft zu versöhnen sucht. Das Meer wird nun nicht länger als jener einsame Raum der deutschen Nation bzw. des deutschen kollektiven Unbewussten inszeniert, sondern als ein gemeinsamer transnationaler Raum, in dem interkulturelle Begegnungen mit dem eigenen und fremden Anderen, mit Kriegsopfern, Alliierten und Gegnern möglich werden: Die italienischen Kriegsgefangenen werden zum Beispiel mit einem anderen deutschen U-Boot an Land befördert, einige Schiffbrüchige mit einem französischen Schiff. Auch die US-amerikanische Armee spielt als Akteurin eine Rolle. Diese Verhandlung und Umgestaltung des Kollektiven wird vor allem aber wegen des Meeres möglich, das im Film aufgrund seiner spezifischen asemantischen Materialität die Symbolisierung der Figuren fördert. 
Zwei Sujetlinien laufen in Laconia aufeinander zu, bis sie in der Katastrophe der Schiffsversenkung vereint werden, sodass die Kollision zugleich auch die der etablierten Bilder und Sinnmuster darstellt: Das englische Schiff Laconia reproduziert die Hollywood-Titanic-Ikonografie (Abb. 11) und andere englisch/amerikanische Darstellungstraditionen, wie etwa Klassendifferenzen sowie puritanische, steife Ladys, feige und grausame Väter, mutige Mütter, einen edlen Kapitän usw. Die Figuren tanzen und singen auf dem Schiff, während sich, in Analogie zu den Unterschichten in Titanic, unter der Wasserlinie auf der Laconia italienische Kriegsgefangene und Vertreter der polnischen Befreiungsarmee befinden, die diese bewachen (Abb. 12).

Abbildung 11: Laconia. In der Darstellung des Schiffes und seiner Versenkung zitiert der Film vor allem Titanic.

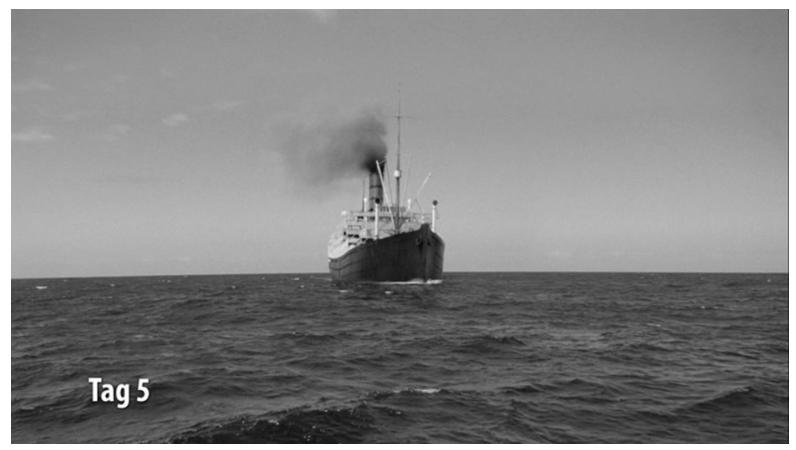

Abbildung 12: Laconia. Ähnlich wie in Titanic werden die Kriegsgefangenen im Bauch des Schiffes eingesperrt.

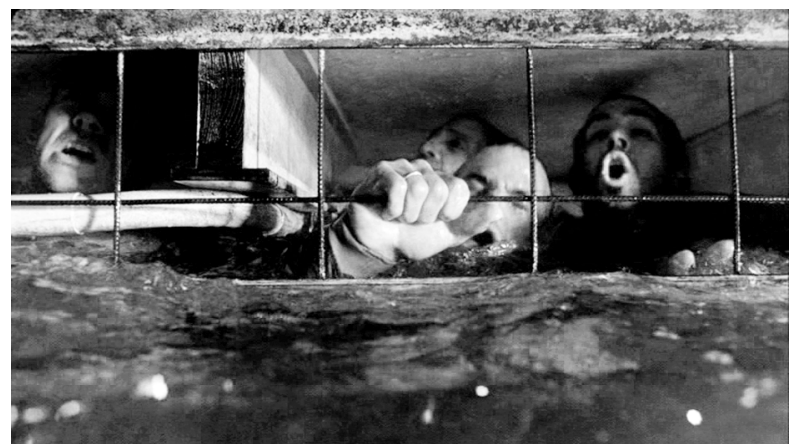

Das deutsche U-Boot ist hingegen Träger einer deutschen Ästhetik, etwa des homoerotischen Männerbundes und der Erzeugung einer gepanzerten Kollektivität. Aus beiden setzt sich dann eine neue EU-Symbolik zusammen. Zuerst werden durch die Spaltung des nationalen Selbst in allen Gruppen Ambivalenzen erzeugt, um den Nationsbegriff und nationale Stereotype aufzulockern: Unter Deutschen (bis auf die Hauptfigur allesamt Männerfiguren), Engländer*innen, 
Italienern, Polen und später US-Amerikanern (ebenfalls jeweils nur Männerfiguren) sind mutige und ehrliche Menschen sowie feige, grausame und regimetreue Personen zu finden; in allen Gruppen gibt es also Täter und Opfer, wobei die binäre Aufteilung von männlichen Soldaten und weiblicher Zivilbevölkerung, von männlichen Tätern und tendenziell weiblichen Opfern auffallend ist. Die Männer in der Zivilbevölkerung werden als feige und egoistisch dargestellt, weshalb sie im Film häufiger Blamagen ausgesetzt sind. Die Zuweisung der kollektiven Schuld läuft also nicht mehr über die Nationalität oder Staatsangehörigkeit, sondern wird geschlechtsspezifisch und situativ erzeugt und so als eine anthropologische Größe verhandelt. Angesichts der Krisensituation zeigt jede*r sein*ihr >wahres< Wesen.

Der zweite Schritt ist die Annäherung an das Andere, die vor allem durch die Erschaffung der transnationalen Erinnerungsfigur Hildegard/Hilda vollzogen wird. Die Nähe der Namen weist auch auf die historische Nähe beider Länder, ja ihren gemeinsamen Ursprung hin, welcher durch die Eltern dieser Figur noch einmal unterstrichen wird: Ihre Mutter ist Engländerin und der Vater Deutscher, wodurch Nationen deessentialisiert werden. Identitätsursprünge fallen nicht mit politischen Staatsgrenzen zusammen. Die Menschen verstehen und vereinigen sich trotz der Sprach-, Kultur- und Ideologiegrenzen. Als deutsche Torpedos das Schiff gegen Ende des ersten Teils beschädigen, treffen sie zugleich das deutsche Publikum selbst, das auch das Leiden des Eigenen und des Anderen, dem sich Hilda Smith angenähert hat, miterlebt. Hier soll die Schuld beglichen und die militärische Aggression gebüßt werden. Mortimer wie auch Hilda Smith verlieren ihre Familien durch die Deutschen. Durch die Torpedos ertrinkt das Baby von Hildas Bruder - da er von den Nazis hingerichtet wurde, hatte sie es in ihre Obhut genommen. Der Tod ihrer letzten Verwandten ist zugleich ein Sinnbild, das affektiv und erinnerungspolitisch wirkt. Diese Szene verweist wieder auf Lifeboat - dort ertrinkt auch ein Baby, woraufhin sich die Mutter das Leben nimmt. Der Krieg trifft zum einen immer die Falschen, wodurch generell Kritik an jeglichem Krieg formuliert wird. So lange Deutschland militärisch aktiv bleibt, wird ihm selbst die Zukunft verwehrt, für die das Kind im Film traditionsgemäß steht (vgl. Edelman 2004). Versehrt und verletzt, sind nun alle Figuren bereit für eine Transgression. Um zu überleben, müssen sie sich von ihren jeweiligen nationalen Identitäten und politischen Positionen lösen.

Der nächste Schritt ist daher die Auflösung der tradierten Ethnizitätsgrenzen. Der Panzerkörper wie die Ströme bekommen jeweils tradierte Gesichter und Körper, um beide Seiten in eine Interaktion zu bringen. Das U-Boot mit der Mannschaft auf dem Deck nähert sich an das Andere an: Zunächst hört der U-Boot-Kapitän Schreie, dann sieht er die Menschen im Meer durch das Fernglas. Diese Szene ist eine Umschreibung der Szene aus U 47 - Kapitänleutnant Prien, bei der keine Personen mehr zu retten waren, und aus dem Film Das Boot, in dem die Mannschaft niemanden retten durfte. Der weibliche Strom verdichtet und materialisiert sich letztendlich in der Figur Hilda Smiths, die sowohl als Schiffbrüchige des englischen Schiffes das Fremde als auch als Deutsche das eigene Andere repräsentiert (Abb. 13-16). 
Abbildungen 13 bis 16: Laconia. Der Körperpanzer und das Andere treffen aufeinander.
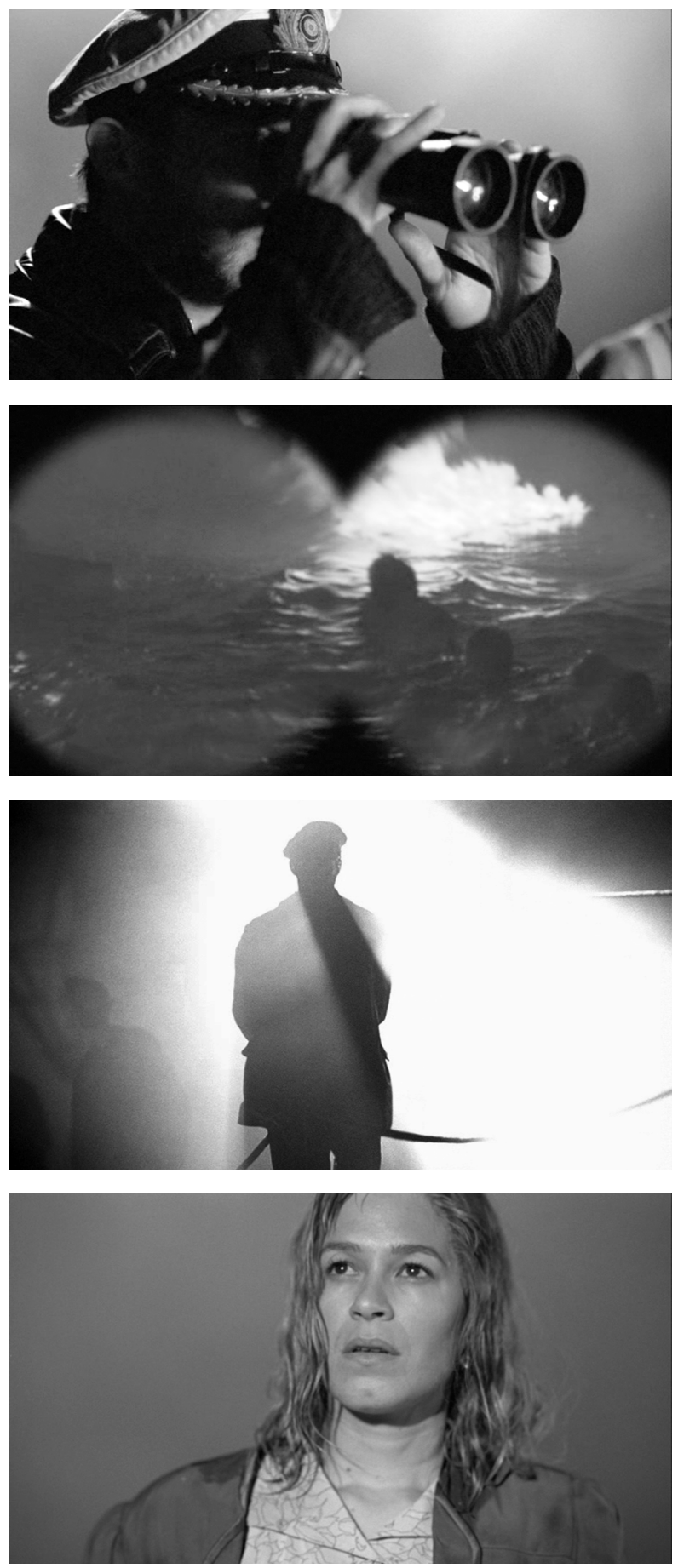
Im weiteren Verlauf betreten alle das U-Boot - die Grenzen bestehen also nicht mehr. Die Kamera durchfährt mehrmals das Innere des U-Bootes, wodurch alle räumlich und visuell zusammengebunden werden. Wenn das innen volle UBoot mit den Menschen an Deck und mit Rettungsbooten von oben gefilmt wird, verschmelzen die Personen visuell zur Einheit (Abb. 4). Hier werden deutsche Soldaten bzw. Matrosen mit den Folgen der eigenen Handlung konfrontiert ein Topos, der im Laufe eines intergenerationellen Wechsels entwickelt worden ist, als ob aus heutiger Perspektive die Chance bestünde, den Tätern gegenüber einen Vorwurf oder eine Anklage auszusprechen. Aber auch umgekehrt können die Anderen erfahren, wie schrecklich es eigentlich ist, ein deutscher Körperpanzer zu sein - die Enge, die Askese und die Gefahren des U-Bootes werden ebenfalls abgebildet.

Vor allem durch die Gefahren wird dann eine neue transnationale Gemeinschaft aus westlichen Ländern geschaffen: Das Probefluten geht schief, das Boot sinkt unkontrolliert weiter, was wiederum symbolisch zu verstehen ist - eine weitere Konfrontation innerhalb der EU schadet allen. Sämtliche Passagier*innen müssen zu einer bestimmten Seite des U-Bootes laufen und so das unkontrollierte Sinken aufhalten. Der Unfall zwingt zur Solidarität aller Anwesenden, und so werden Engländer*innen, Italiener und Deutsche zu einer Gemeinschaft von Menschen, die aus dieser existenziellen Erfahrung der Bedrohung und Konfrontation heraus geboren wird (Abb. 17).

Abbildung 17: Laconia. Nur gemeinsam können die Figuren das U-Boot vor dem Versinken retten. Auch die bildliche Strategie fasst sie als transnationales Kollektiv - sie sind zudem glücklich.

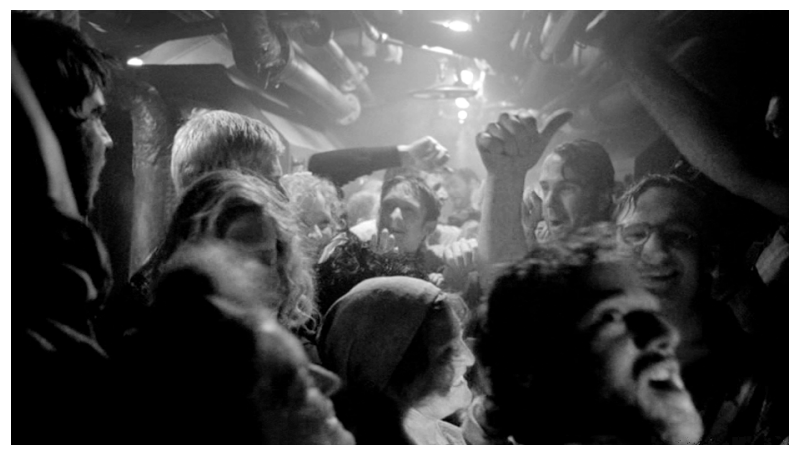

In dieser neuen Weltordnung sind vor allem die Polen nicht willkommen - sie gehören in diesem Film zu den Tätern, wobei Polen eher als der Repräsentant Osteuropas an sich zu verstehen ist, das negativ bewertet wird. Zum einen ist die Erweiterung Europas nach Osten offensichtlich nicht so sehr erwünscht oder eher gefürchtet. Zum anderen geht es auch um die Gegenwartspolitik, in der Polen und Osteuropa sich generell nicht mit den westlichen EU-Ländern solidarisieren. Als der größte Geldgeber in der EU retten die Deutschen am Ende alle, 
während Großbritannien als Kolonialstaat nur für die eigenen Interessen arbeitet und die Amerikaner die Schiffbrüchigen bombardieren. Die USA zeigen sich als militärisch aggressiv, was ebenfalls als Kommentar auf die imperiale US-Politik der Gegenwart zu deuten ist.

Das Meer, so lässt sich abschließend zusammenfassen, bedingt eine besondere Ästhetik, in der kollektive, tradierte und unbewusste Symbole neu ausgehandelt werden können. Laconia schreibt die Vergangenheit um und zielt vor allem darauf ab, eine überzeugende kollektive EU-Symbolik zu entwickeln, welche aufgrund der zum Einsatz gebrachten generischen und ästhetischen Traditionen und durch die Inszenierung eines >authentischen $<$ historischen Falls tatsächlich eine große Wirkung entfaltet. Die Signifikation vollzieht dabei eine rekursive Bewegung: Aus der Gegenwart heraus wird die Vergangenheit neu erschaffen, welche wiederum auf die Gegenwart zurückwirkt und die neue politische Ordnung aus der Kraft der (Film-)Geschichte heraus legitimiert.

\section{LITERATUR}

Assmann, Jan (2006): Das gerettete Wissen. Flutkatastrophen und geheime Archive. In: Ders./Martin Muslow (Hg.): Sintflut und Gedächtnis. Erinnern und Vergessen des Ursprungs. München, S. 291-301.

Blair, Clay (1999): Der U-Boot-Krieg. Bd. 2: Die Gejagten, 1942-1945. München.

Bruns, Claudia (2009): Europas Grenzdiskurse seit der Antike - Interrelationen zwischen kartographischem Raum, mythologischer Figur und europäischer Identität. In: Michael Gehler/Andreas Pudlat (Hg.): Grenzen in Europa. Hildesheim, S. 17-64.

Butler, Judith (2010): Raster des Krieges. Warum wir nicht jedes Leid beklagen. Aus dem Engl. v. Reiner Ansén. Frankfurt a.M.

Csáky, Moritz/Leitgeb, Christoph (Hg.; 2009): Kommunikation - Gedächtnis - Raum: Kulturwissenschaften nach dem "Spatial Turn«. Bielefeld.

Döring, Jörg/Thilmann, Tristan (Hg.; 2009): Spatial Turn. Das Raumparadigma in den Kultur- und Sozialwissenschaften. Bielefeld.

Edelman, Lee (2004): No Future: Queer Theory and the Death Drive. Durham.

Elias, Norbert ( $\left.{ }^{16} 1991\right)$ : Über den Prozeß der Zivilisation. Soziogenetische und psychogenetische Untersuchungen. Bd. 1: Wandlungen des Verhaltens in den weltlichen Oberschichten des Abendlandes. Frankfurt a.M.

Elsaesser, Thomas (2006): Geschichte(n) und Gedächtnis. Zur Poetik der Fehlleistungen im Mainstreamkino am Beispiel von "Forrest Gump". In: Irmbert Schenk u.a. (Hg.): Experiment Mainstream? Differenz und Uniformierung im populären Kino. Berlin, S. 31-42.

François, Etienne/ Schulze, Hagen (Hg.; 2001): Deutsche Erinnerungsorte. 3 Bde. München.

Grob, Norbert (2010): Kammern unter Wasser. Sieben Anmerkungen zum U-Bootfilm. In: Roman Mauer (Hg.): Das Meer im Film. Grenze, Spiegel, Übergang. München, S. 223-237. 
Heller, Heinz/Röwekamp, Burkhard/Steinle, Matthias (Hg.; 2007): All Quiet on the Genre Front? Zur Praxis und Theorie des Kriegsfilms. Marburg.

Horn, Eva (2007): Der geheime Krieg. Verrat, Spionage und moderne Fiktion. Frankfurt a.M.

Kamps, Johannes (2006): 100 U-Boot-Filme. In: Deutsches Filmmuseum Frankfurt (Hg.): "Das Boot auf der Suche nach der Crew der U96“. Katalog zur gleichnamigen Ausstellung. Frankfurt a.M., S. 182-197.

Klein, Thomas/Stiglegger, Marcus/Traber, Bodo (Hg.; 2006): Filmgenres: Kriegsfilm. Stuttgart.

Koldau, Linda Maria (2012): Submarine Films as Aesthetic Reflection of War History and War Strategy. In: Søren R. Fauth / Kasper Green Kreijberg/Jan Süselbeck (Hg.): Repräsentationen des Krieges. Emotionalisierungsstrategien in der Literatur und in den audiovisuellen Medien vom 18. bis zum 21. Jahrhundert. Göttingen, S. 102118.

Krützen, Michaela (2004): Dramaturgie des Films: Wie Hollywood erzählt. Frankfurt a.M. Mauer, Roman (2010): Einleitung. In: Ders. (Hg.): Das Meer im Film. Grenze, Spiegel, Übergang. München, S. 9-28.

Nora, Pierre (Hg.; 2005): Erinnerungsorte Frankreichs. Aus dem Franz. v. Michael Bayer. München.

Ritzer, Ivo (2010): Heterotopisches, Abjektes, Apokalyptisches. Submarine Welten im Film. In: Roman Mauer (Hg.): Das Meer im Film. Grenze, Spiegel, Übergang. München, S. 268-280.

Rosner, Heiko (1981): Wahnsinn des Krieges ehrlich vermittelt. Interview mit dem Regisseur des Films "Das Boot". In: Kreiszeitung Wesermarsch v. 18. September 1981.

Sabrow, Martin (Hg.; 2009): Erinnerungsorte der DDR. München.

Theweleit, Klaus (1977): Männerphantasien. Bd. 1: Frau, Fluten, Körper, Geschichte. Frankfurt a.M.

Völger, Gisela/Welck, Karin von (Hg.; 1990): Männerbande - Männerbünde. Zur Rolle des Mannes im Kulturvergleich. 2 Bde. Köln.

Weigel, Sigrid (2002): Zum "topographical turn". Kartographie, Topographie und Raumkonzepte in den Kulturwissenschaften. In: KulturPoetik. Zeitschrift für kulturgeschichtliche Literaturwissenschaft 2, H. 2, S. 151-165.

Widdig, Bernd (1997): ,Ein herber Kultus des Männlichen: Männerbünde um 1900. In: Walter Erhart/ Britta Herrmann (Hg.): Wann ist der Mann ein Mann? Zur Geschichte der Männlichkeit. Stuttgart/Weimar, S. 235-248.

Winkler, Willi (2011): Vom hilfreichen Feind. In: SZ.de, 2. November 2011; online unter: http://www.sueddeutsche.de/medien/kriegsfilm-laconia-in-der-ard-vom-hilfreichen-feind-1.1178591 [Stand: 1.10.2020].

Wolf, Burkhardt (2013): Fortuna di mare. Literatur und Seefahrt. Zürich/Berlin. 